\title{
RP-Sim: Radio Propagation Simulator
}

\section{Matheus Ferraroni Sanches, Oscar Jaime Ciceri Coral, Leandro Aparecido Villas}

\author{
${ }^{1}$ Instituto de Computação - Universidade Estadual de Campinas (UNICAMP) \\ Cidade Universitária Zeferino Vaz - Barão Geraldo, Campinas - SP, 13083-970 \\ \{ferraroni, oscar, leandro\}@lrc.ic.unicamp.br
}

\begin{abstract}
Radio Propagation Simulator (RP-Sim) is a tool that was developed with the purpose of assisting in the analysis of coverage and performance of wireless networks, assisting during the planning stage for and allocation of infrastructure. In its first version, to calculate the reception power and bit error rate (BER) in a region or in a specific location, RP-Sim is already able to consider obstacles, such as buildings and constructions, different propagation models, different modulations and configuration of the communication devices, which include positioning, height, gains, frequency and bandwidth. In addition, $R P$-Sim allows to export communication regions that reach a maximum BER value or a minimum reception power.
\end{abstract}

Resumo. Radio Propagation Simulator (RP-Sim) é uma ferramenta que foi desenvolvida com o propósito de auxiliar na análise de cobertura e desempenho de redes sem fio, podendo auxiliar durante o estágio de planejamento e alocação de infraestrutura. A versão atual do RP-Sim considera obstáculos, como prédios $e$ construções, diferentes modelos de propagação, diferentes modulações $e$ configuração dos dispositivos de comunicação, como posicionamento, altura, ganhos, frequência e largura de banda, para calcular a potência de recepção e a taxa de erro de bit (BER) em uma região ou em um local específico. Além disso, RP-Sim permite exportar regiões de comunicação que atinjam um valor máximo de BER ou uma potencia de recepção mínima.

\section{Introdução}

A realização de planejamento e a implantação de infra estrutura para prover comunicação com dispositivos sem fio são tarefas complexas e têm recebido cada vez mais parâmetros relacionados ao cenário e a comunicação. Considerar informações relacionadas a configuração de comunicação entre dispositivos, obstáculos, modulação e modelos de propagação, são tarefas que requerem tempo e podem ser uma barreira para quem lida com isso. Ao desconsiderar tais informações, a complexidade da tarefa pode ser reduzida, mas, ao mesmo tempo, pode distanciar os resultados obtidos nas simulações em comparação com os resultados obtidos no mundo real.

Levar em consideração os limites presentes nessa situação é essencial para uma boa alocação e para o bom funcionamento dos serviços que dependem dessa comunicação. Os dados sobre potência de recepção e BER em diferentes locais são importantes para que os objetivos de cobertura definidos sejam atingidos de maneira eficiente.

O conhecimento detalhado da propagação das ondas de rádio nas cidades é indispensável para fazer o planejamento adequado da alocação de infra estrutura. Para isso, 
foi desenvolvido o Radio Propagation Simulator (RP-Sim ), ferramenta capaz de considerar diversas informações relevantes para essa tarefa e automatizar o processo de cálculo da propagação de sinal em diferentes ambientes, como urbanos e rurais. A ferramenta proposta também permite simular a propagação de sinal considerando as construções da região, o modelo de propagação de sinal e a modulação que mais se adéquem a necessidade. Além disso, a ferramenta também permite exportar as áreas onde a comunicação atinja valor mínimo de potência recebida ou máximo de BER. A ferramenta foi desenvolvida para ser utilizada de maneira fácil e rápida, até mesmo por pessoas com pouca experiência na área, auxiliando, assim, estudantes, professores, pesquisadores e empresas.

Com o objetivo de facilitar o uso, toda a ferramenta RP-Sim foi feita completamente com código aberto e planejada para ser utilizada direto pelo navegador do usuário, necessitando apenas de acesso com a internet para carregar o mapa. A arquitetura utilizada foi pensada para permitir que os pesquisadores e futuros usuários consigam criar suas próprias melhorias e implementar novos modelos de propagação e modulação do sinal, permitindo que sua utilização seja cada vez mais abrangente. Por conta de sua flexibilização, também é possível avaliar novas tecnologias e o impacto delas nos serviços e aplicativos relacionados a comunicação sem fio. Com a utilização do RP-Sim esperamos que a academia e a indústria possam encontrar soluções melhores, mais rápidas, mais baratas e com menos esforços, beneficiando a todos neste ciclo.

O código fonte, descrições adicionais e tutorial passo a passo estão disponíveis no endereço https://github. com/MatheusFerraroni/RP-Sim. Um vídeo demonstrando a utilização da ferramenta está disponível em https: / / www • youtube . $\mathrm{com} /$ watch?v=z59Plq7Yw34. Para utilizar a ferramenta, uma versão foi disponibilizada em https://matheusferraroni.github.io/RP-Sim/, já que sua utilização pode ser feita sem instalação. A distribuição será feita com a licença $G N U$ Affero General Public License v3.0 (AGPL-3.0), por ser uma licença bastante permissiva, que concede o direito de alterar o código fonte de acordo com a necessidade dos usuários, desde que eles compartilhem suas alterações e mantenham a mesma licença.

\section{Arquitetura}

A arquitetura desenvolvida para o RP-Sim é apresentada na Figura 1, onde são apresentadas três principais divisões da ferramenta. A parte 'Mapa e Interação' é responsável por apresentar o mapa e controlar as interações com o mesmo. Na parte 'RP-Sim' estão os módulos do RP-Sim, bem como o cálculo da propagação do sinal e a gerência das construções e antenas. A parte 'Visualização' é responsável por apresentar os resultados obtidos de maneira visual sobre o mapa.

O mapa Leaflet ${ }^{1}$ foi utilizado por ter seu código aberto, por ser fácil de utilizar pelos usuários finais e por ter carregamento rápido e ser leve. Esse mapa já está preparado para ser utilizado em dispositivos móveis.

A parte 'RP-Sim' é responsável por realizar os processamentos necessários para as funcionalidades desenvolvidas. Essa parte está dividida em 7 módulos que são responsáveis desde o controle dos pontos amostrais, até a realização dos cálculos das intersecções da comunicação entre antena e ponto amostral. Os módulos da ferramenta são descritos abaixo:

\footnotetext{
${ }^{1}$ https://leafletjs.com/
} 


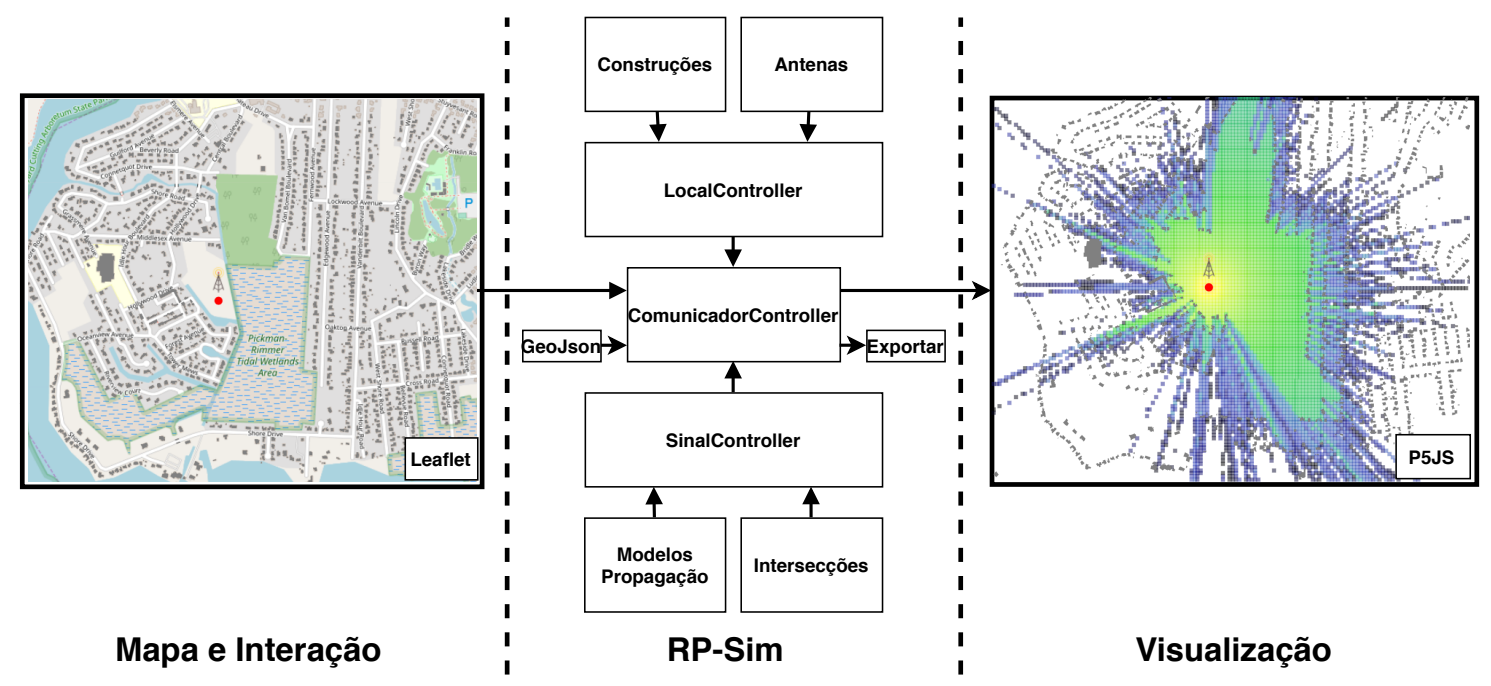

Figura 1. Arquitetura RP-Sim

1. Construções: Informações sobre formato e altura para cada construção são salvas neste módulo. Funções de criar, gerenciar e mostrar as construções no mapa, também estão implementadas aqui.

2. Antenas: Esse módulo é responsável pela gerência das antenas, guardando informações como posição e sendo responsável pela sua apresentação no mapa.

3. LocalController: Esse módulo é responsável por mapear os pontos amostrais onde o RSSI e o BER serão calculados. A visualização dessas informações são definidas aqui.

4. Intersecções: Esse módulo é responsável por calcular a interseção entre a linha formada pela antena e ponto amostral com as construções do cenário. Foi criado de forma separada, pois a posição de checagem de RSSI muda de acordo com a granularidade de checagem utilizada, que se ajusta automaticamente com o zoom no mapa.

5. Modelos Propagação: São classes responsáveis por definir a propagação do sinal. Os modelos de propagação implementados na ferramenta são:

- Sem obstáculos

- SUI [Anusha et al. 2017]

- Egli [Egli 1957]

- Ericsson [Chariyev et al. 2014]

- Nakagami-m [Beaulieu and Cheng 2005]

- Free Space [Friis 1946]

- Okomura Hata [Nadir and Ahmad 2010]

- Two Ray Ground [Sommer et al. 2012]

- Fixed Distance

- Obstáculos

- Por metro em obstáculos [Sommer et al. 2011]

- Por corte no sinal

- Por corte causado por obstáculos [Sommer et al. 2011]

Também considera-se a modulação da comunicação para definir o BER [Meghdadi 2008]. As modulações implementadas na ferramenta são: BSPK, QPSK, 8PSK, 16PSK, 4QAM, 16QAM e 64QAM 
6. SinalController: As configurações de frequência, largura da banda, potência de transmissão, ganhos, altura de transmissão e recepção e qual modulação está selecionada, ficam salvas nesse módulo para serem convertidas para as medidas necessárias. Qual modelo de propagação está ativo e sua execução também são responsabilidades desse módulo.

7. ComunicadorController: Esse módulo é responsável pela integração de todos os outros. Responsável, efetivamente, por calcular a propagação do sinal entre a posição das antenas e do locais definidos para checagem, considerando as informações disponíveis e configurações definidas. Também implementa as funções de importação e exportação dos dados necessários.

A terceira parte é responsável por apresentar os resultados de maneira visual. Essa apresentação é feita com uma sobreposição da camada de visualização sobre o mapa, facilitando o entendimento. Para colocar as informações necessárias na camada de visualização, foi utilizada a biblioteca Mappa.js ${ }^{2}$ em conjunto com a biblioteca P5JS ${ }^{3}$. A biblioteca Mappa.js se encarrega da alinhar a sobreposição da camada de visualização com o mapa e a biblioteca P5JS permite que os resultados obtidos sejam apresentados de maneira simples e rápida.

\subsection{Implementação}

Para realizar a implementação da ferramenta foram adotadas tecnologias WEB, principalmente a linguagem de programação Javascript, por ser a linguagem mais popular de 2014 a 2019 [GitHub 2019]. A utilização dessa linguagem também permite acesso a avançados recursos visuais e inúmeras bibliotecas que podem auxiliar na evolução da ferramenta. Com a utilização de tecnologias WEB, é possível que a execução aconteça diretamente do navegador do usuário, facilitando o acesso e removendo requisitos como instalação de programas no dispositivo. A ferramenta também foi desenvolvida de maneira que não seja necessário a utilização de servidores dedicados para seu funcionamento, apenas o computador por onde ela foi acessada.

A Figura 2 apresenta o diagrama de classes que foi implementado para as classes de propagação de sinal. É possível ver que existem 3 especializações distintas para a propagação de sinal: 'PropagationCorte', que considera o número de vezes que o sinal foi interrompido; 'PropagationObstacle', que considera a distância percorrida dentro de obstáculos; e 'PropagationNoObstacle', que considera a distância percorrida fora de obstáculos.

Pelo diagrama apresentado, é possível observar que a implementação de um novo modelo pode ser feita de maneira simples, apenas especializando uma das 3 principais classes e implementando o método 'calculate'. O método 'calculate' recebe sempre 3 parâmetros: a distância percorrida fora de obstáculos em metro; a distância percorrida dentro de obstáculos em metros; e a quantidade de vezes que o sinal foi interrompido por obstáculos. Para implementar o modelo de propagação 'FreeSpace', por exemplo, basta somar as distâncias de interesse, dividir por 1000 para transformar em quilômetros, aplicar a fórmula do FreeSpace e retornar o valor obtido.

\footnotetext{
${ }^{2}$ https://mappa.js.org/

${ }^{3}$ https://p5js.org/
} 


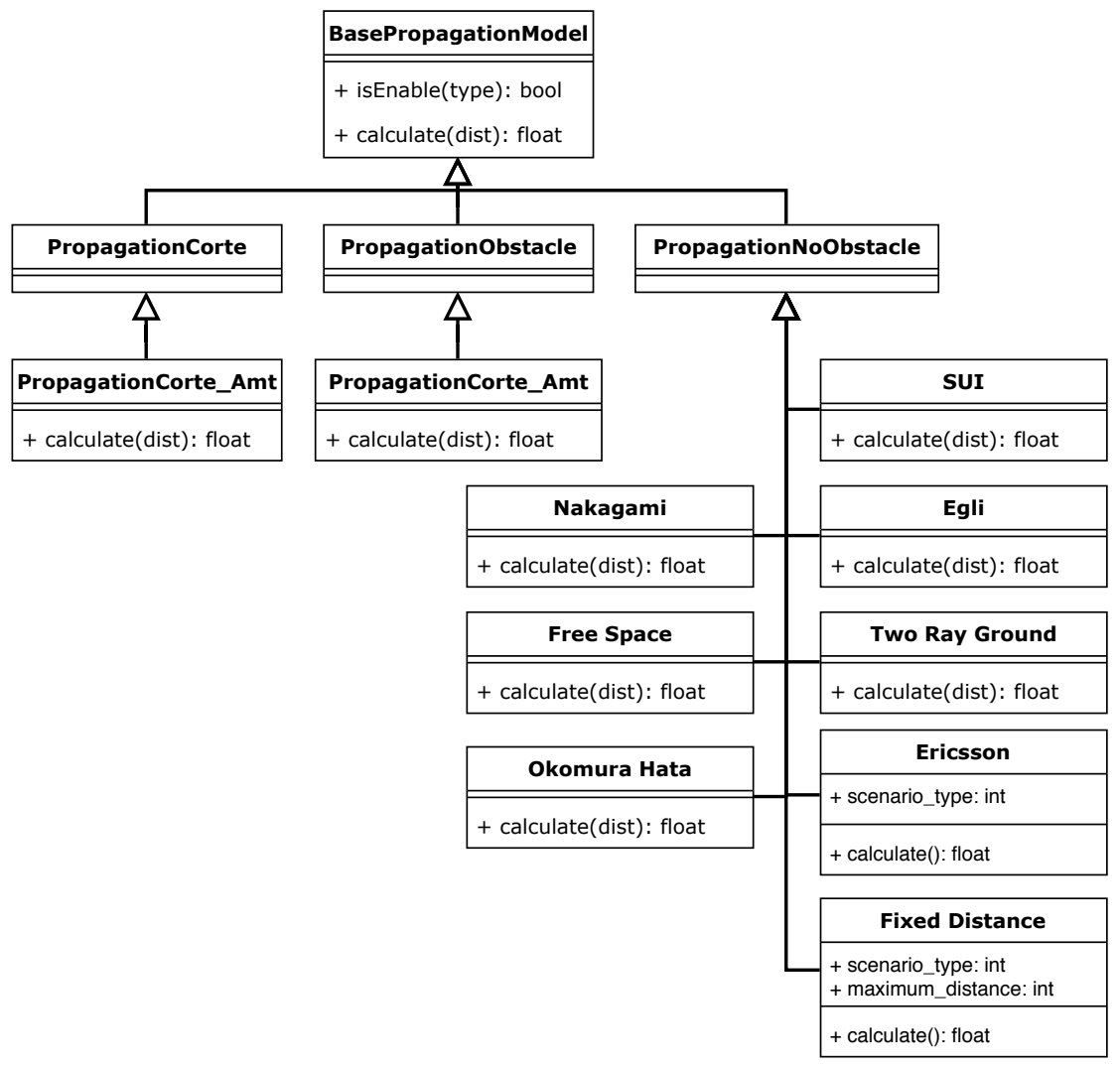

Figura 2. Classes de propagação de sinal

Quando configurações específicas para um novo modelo de propagação são necessárias, é possível adaptar o código que insere as informações nas configurações dos modelos de propagação para que campos especiais sejam adicionados.

\subsection{Entrada}

O padrão de inserção de dados externos é o GeoJson, que, atualmente, é utilizado para carregar as localizações para as antenas e carregar dados, como formato e posição dos obstáculos. O GeoJson é um formato para armazenar informações geográficas utilizando Json. A obtenção dos dados sobre os obstáculos pode ser feita utilizando a ferramenta Overpass Turbo ${ }^{4}$, que consegue requisitar de maneira rápida e fácil os dados do OpenStreetMaps.

\subsection{Saída}

A saída de dados foi padronizada no formato GeoJson, por ser um formato amplamente conhecido e utilizado. O principal dado exportado na ferramenta são os polígonos da área de comunicação com as antenas. Esses polígonos são definidos de acordo com as configurações que foram fornecidas, como frequência e potência de transmissão, e o usuário pode definir qual o valor mínimo de Received Signal Strength (RSSI) ou valor máximo para o BER. Durante o estágio de exportação o RSSI mínimo é apresentado e o valor do BER esperado para o RSSI selecionado, assim é possível alterar o RSSI mínimo

\footnotetext{
${ }^{4}$ https://overpass-turbo.eu/
} 
para ser compatível com o BER máximo aceitado. Os polígonos exportados são posições no padrão de coordenadas WGS84, que é o mais utilizado em sistemas geográficos.

\section{Funcionalidades}

A ferramenta desenvolvida compreende diversos módulos para calcular a propagação do sinal, o RSSI recebido e o BER, de acordo com o RSSI. Para isso, foram implementadas funções para importação e exportação de dados, classes para propagação de sinal e diversos controles e configurações, que permitem utilizar a ferramenta para as mais diversas finalidades que estejam relacionadas com o cálculo da propagação de sinal.

A parte de visualização dos resultados obtidos foi montada para funcionar em conjunto com o mapa, já que os dados gerados são todos geo-referenciados. Uma camada de visualização foi adicionada sobre o mapa e as informações foram apresentadas de maneira intuitiva em conjunto com diferentes escalas de cores. É possível visualizar a propagação do sinal, o RSSI recebido em diferentes locais, o BER calculado de acordo com o RSSI e mapear áreas com uma qualidade mínima de sinal ou de RSSI.

Para determinar o RSSI recebido e o BER, são consideradas as seguintes configurações: temperatura; altura da antena transmissora; altura da antena receptora; figura de ruído; frequência de comunicação; largura do canal; ganho de transmissão; ganho de recepção; diferentes modelos de propagação de sinal e diferentes modulações para a comunicação. Além disso, um ponto importante considerado são os módulos específicos para definir o impacto causado pelos obstáculos no cenário.

Além das funcionalidades apresentadas na arquitetura, as classes de propagação de sinal foram projetadas de maneira que evoluções, adaptações e melhorias, possam ser feitos de maneira simples e rápidas, incentivando o uso da ferramenta pela comunidade, que pode adaptá-la às suas necessidades.

\section{Demonstração}

A utilização da ferramenta foi idealizada para ser o mais simples possível, para permitir que até mesmo pessoas com pouca experiência consigam fazer o uso. Para isso, configurações básicas e pré configuradas já são carregadas junto com a ferramenta.

A utilização foi definida em passos simples e diretos. Entre os passos 3 e 4 é possível adicionar um passo extra para definir as configurações para a comunicação e ambiente, para que os cálculos se aproximem o máximo possível da situação desejada. Sua utilização depende apenas de um computador com navegador e acesso à internet.

1. Abrir o site do RP-Sim

2. Importar arquivo com posições e formatos das construções

3. Importar arquivo com as posições das antenas

4. Calcular

Os cálculos de intersecção da linha de comunicação, entre a antena transmissora e a receptora, é um processo mais lento, por isso cria-se um cache na primeira execução. Assim, sempre que alguma configuração for alterada, exceto a posição dos obstáculos, das antenas, ou o zoom, os cálculos são refeitos de maneira quase instantânea, assumindo que a intersecção já está calculada. O zoom também tem um papel fundamental nesse 
cache, devido à sua utilização para definir automaticamente a granularidade dos locais para realizar a amostragem do RSSI e do BER.

A Figura 3 apresenta um exemplo da ferramenta sendo utilizada para visualizar a propagação do sinal. As cores no mapa indicam o RSSI sendo recebido por cada local avaliado. As configurações nesse teste representam a alocação de 2 RoadSide Units em uma região com poucas construções.

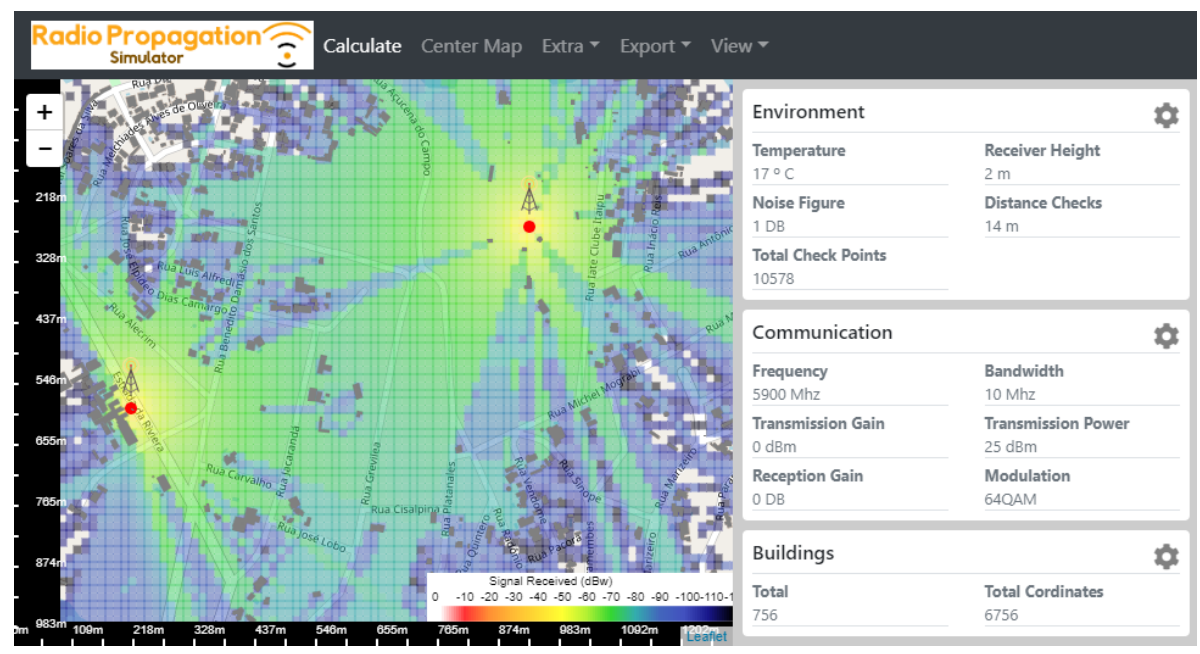

Figura 3. Exemplo de RSSI resultado obtido

Uma vez que o processo de calcular o RSSI recebido esteja completo é possível trocar a visualização para o modo BER, apresentado na Figura 4, e para o modo onde são gerados polígonos de qualidade de sinal, apresentado na Figura 5.

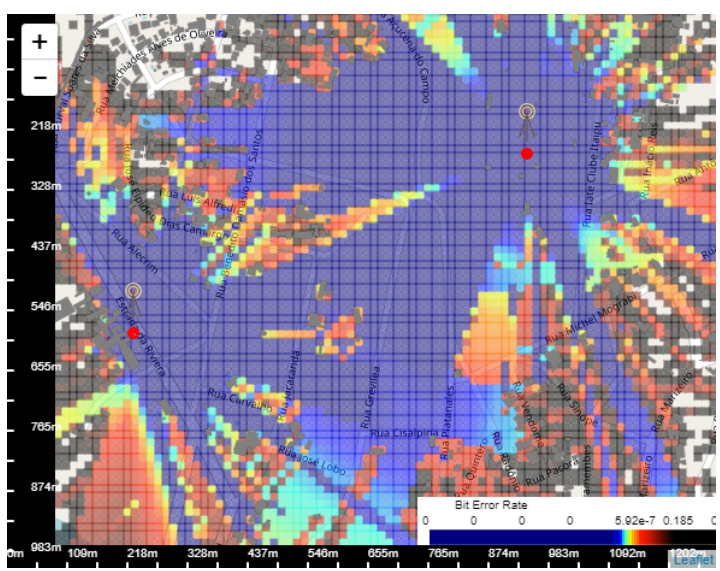

Figura 4. BER

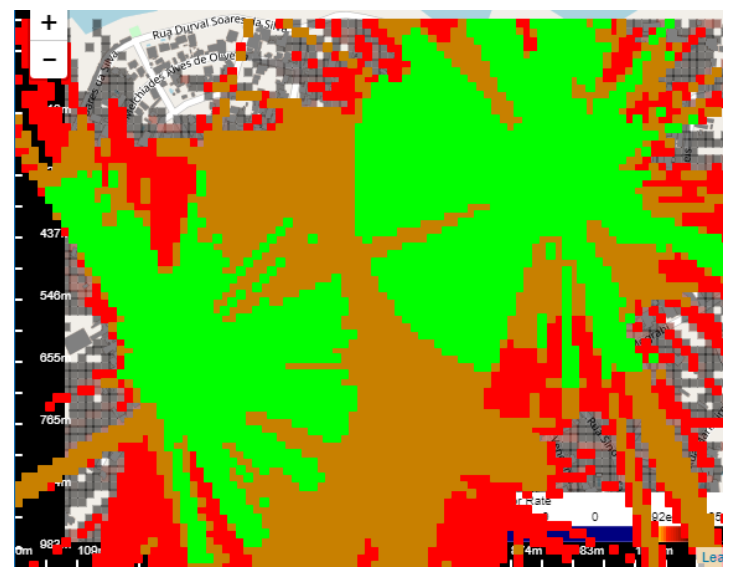

Figura 5. Área vs RSSI mínimo

Os resultados obtidos na Figura 5 podem ser exportados no formato geojson, contendo os 3 polígonos que contém a área de comunicação com sinal considerado bom, médio e ruim. É possível alterar o valor mínimo de RSSI e o BER máximo para que uma área seja considerada boa, média ou ruim. 


\section{Conclusão e Trabalhos Futuros}

Este trabalho apresentou a ferramenta Radio Propagation Simulator (RP-Sim). Tal ferramenta é inteiramente WEB, sem a necessidade de servidores dedicados para auxiliar alunos, professores, pesquisadores e empresas, a entender a propagação de sinal e auxiliar na alocação de dispositivos para comunicação.

São consideradas as configurações de comunicação fornecidas pelo usuário, obstáculos, como prédios e construções, a posição das antenas, diferentes modelos de propagação de sinal e diferentes modulações para definir regiões onde é possível se comunicar ou não com as antenas.

Para evolução futura, será considerada a quantidade de dispositivos por região e o impacto que isso causa na comunicação. Também será atualizado o formato de propagação do sinal das antenas, sendo possível ter antenas não omnidirecionais.

\section{Referências}

Anusha, V. S., Nithya, G. K., and Rao, S. N. (2017). A comprehensive survey of electromagnetic propagation models. In 2017 International Conference on Communication and Signal Processing (ICCSP), pages 1457-1462.

Beaulieu, N. C. and Cheng, C. (2005). Efficient nakagami-m fading channel simulation. IEEE Transactions on Vehicular Technology, 54(2):413-424.

Chariyev, A., Low, T., and MOHAMAD SAAD, M. N. (2014). Path loss simulation in different radio propagation models with $1.8 \mathrm{ghz}$ and $2.6 \mathrm{ghz}$ bands. In ICCOINS, pages $1-5$.

Egli, J. J. (1957). Radio propagation above $40 \mathrm{mc}$ over irregular terrain. Proceedings of the IRE, 45(10):1383-1391.

Friis, H. T. (1946). A note on a simple transmission formula. Proceedings of the IRE, 34(5):254-256.

GitHub (2019). The state of the octoverse - the state of the octoverse celebrates a year of building across teams, time zones, and millions of merged pull requests. https: // octoverse.github.com/. Acessado: 2019-12-24.

Meghdadi, V. (2008). Ber calculation. Wireless Communications.

Nadir, Z. and Ahmad, M. (2010). Pathloss determination using okumura-hata model and cubic regression for missing data for oman. Lecture Notes in Engineering and Computer Science, 2181.

Sommer, C., Eckhoff, D., German, R., and Dressler, F. (2011). A computationally inexpensive empirical model of ieee 802.11 p radio shadowing in urban environments. In 2011 Eighth International Conference on Wireless On-Demand Network Systems and Services, pages 84-90.

Sommer, C., Joerer, S., and Dressler, F. (2012). On the applicability of two-ray path loss models for vehicular network simulation. In 2012 IEEE Vehicular Networking Conference (VNC), pages 64-69. 